\title{
Investigations of Adhesive Properties of Polyamide Modified with Friction Reducing Agents
}

\author{
Kamil Anasiewicz ${ }^{1 *}$, Józef Kuczmaszewski \\ 1 Lublin University of Technology, Department of Fundamentals of Production Engineering, Nadbystrzycka 36, \\ 20-618 Lublin, Poland \\ * Corresponding author's e-mail: k.anasiewicz@pollub.pl
}

\begin{abstract}
Polyamide is a material used in various industrial sectors. In moving connections, in order to reduce friction between elements made of polyamide, lubrication is used or other substances reducing friction are added, such as molybdenum disulfide. It can be hypothesised that improving the sliding properties of polyamide will adversely affect polyamide's ability to form effective adhesive bonds. An interesting question is whether a possible unfavourable change in adhesive properties of such modified polyamide will significantly influence the strength of adhesive bonds of this material. This paper analyses the adhesive properties of polyamide with respect to the strength of adhesive bonds. In order to verify the hypothesis, comparative tests of tensile shear strength of bonded doubleoverlap samples were carried out. Tests were conducted in accordance with ASTM D3528. Samples were prepared using Polyamide PA6, PA6 - MoS , PA6G + oil and EN AW 2024 aluminium alloy. The following technologies were used to prepare the surface prior to bonding: plasma, sol-gel and abrasive jet machining. The summary of the article indicates that adding friction reducing agents to polyamide does not significantly affect the deterioration of its adhesive properties. Samples prepared with sandblasting achieved form $81.8 \%$ up to $114 \%$ higher shear strength than samples prepared with plasma and sol-gel treatments, disregard of added friction reducing agents to the adhered polyamide material. It is also interesting information of a utility character. A general conclusion can be formulated that the appropriate preparation of the polyamide surface, regardless of its material modification with friction reducing agents, in the technologies analysed, particularly by sandblasting, can ensure satisfactory results of joining the polyamide by adhesive bonding.
\end{abstract}

Keywords: adhesive bonds, surface preparation, polyamide, modification of surface layer.

\section{INTRODUCTION}

Polyamide is an important construction material, especially in the engineering industry. In addition to machine components, it is also used in kinematic nodes as a pivot-bush motion joint or as a motion association of planar surfaces. In modern structures stationary bonds of adhesive nature, especially adhesive bonds, are also very often used in assembly processes. In order to reduce friction between elements made of polyamide in motion joints, oiling or addition of other substances that reduce friction is used, for example molybdenum disulfide or lubricant - this modification is used at the processing stage [1,2].
Such modification may significantly alter its adhesive properties, i.e. the ability to form an adhesive bond of structural character, i.e. prepared to carry mechanical loads of defined values.

Polyamide as a construction material is available in many grades, as basic and modified. Modification makes it possible to obtain many structurally significant features, such as: a large range of variability in terms of stiffness, appropriate hardness, durability and mechanical strength, shape stability in operating conditions, good sliding properties and resistance to abrasion, very good electrical insulation, high vibration damping capacity and impact strength, high resistance to UV radiation, good machinability, chemical 
resistance to oils, fats, greases, petrol, low thermal expansion [3]. These properties make polyamides used for manufacturing such machine elements as slide bearings, wheel caps, spoilers, tank covers, elements of slides and guides, conveyor rollers, wheel and pulley bushings, pulleys, sprockets and gears, cams, hammers, sealing and thrust rings, pull screws, ropes, etc. [4]. Such a wide range of applications makes it necessary to use various methods of joining polyamide elements in mechanical engineering, including those where the phenomenon of adhesion determines the effectiveness of the joint. This applies in particular to bonding, structure sealing and coating.

In bonding, it is particularly important to ensure that the surfaces of the parts to be bonded are properly prepared. A properly prepared surface for bonding is characterised by high cleanliness, good adhesive wetting, ability to form interfacial bonds, stability for the assumed conditions and joint operation time $[5,6]$.

Adhesion can be aided by removing unwanted layers from bonded surfaces, creating a new active surface by coating primers, and changing the surface activity $[7,8]$. These changes can be induced by mechanical methods, chemical (etching), and radiation: UV, X-ray, laser, electron beam, reactive gas exposure (e.g. ozonation, fluorination), and low-temperature plasma exposure, corona discharge or flame treatment $[9,10]$. This means that by appropriate processing, the adhesive properties of materials that are difficult to bond can be significantly improved [11].

Research works presented in the literature very often refer to the physical or chemical properties of the polyamide material itself. Due to the fact that polyamide is common in industry, it is frequently modified by changing its physical or chemical properties $[12,13]$. The literature lacks detailed studies focused on the potential of modified polyamide to form adhesive bonds. Nevertheless, few studies were found focusing on the modification of the surface layer in order to improve its adhesive properties. The conducted research shows the improvement of the adhesive properties as a result of plasma treatment the polyamide surface [7]. The studies presented in the literature also confirm the positive effect of plasma treatment on the wettability of the polyamide surface and the strength of its adhesive bonds [14]. Polyamide is inherently difficult to bond. There are also research which discusses various methods of surface preparation for the fatigue strength of the adhesive joint were analysed. However, they usually referred to the basic polyamide PA6 not modified in any way [15]. Another research discusses the laser treatment of the surface of polyamide CFRP composite. From the results of these studies it can be concluded that the laser treatment significantly improved the strength of adhesive joints made on surfaces prepared in this way [16]. The research on the treatment of helium-dielectric discharge on the adhesive properties of the PA6 polyamide surface should be cited as well. This type of treatment strongly develops the geometric surface of the polyamide, which also favours the formation of strong adhesive bonds [17]. Despite the works presented in the literature, no studies have been found that would deal with the subject of joining by adhesive bonding a modified polyamide solely to work in motion joints - with a reduced coefficient of friction. As already mentioned, modification of the polyamide - not its surface itself, can potentially adversely affect the strength of the adhesive bonds made by joining the modified polyamide.

The problem of bonding of modified polyamides in the aspect of friction factor reduction is particularly interesting from the scientific and practical point of view. Such modification may result in a decrease in the surface free energy. This is a disadvantageous situation from the perspective of efficiency of wetting such a material by the adhesive, and consequently reduction of strength of the joint. In the reviewed literature, there are not many works on structural bonding of polyamide components $[14,18]$. Generally, thermoplastics, due to their chemical structure and physical properties, especially bending and tensile stiffness, are difficult in adhesive bonding [10]. An important issue in these situations is to use the appropriate technology, especially surface preparation technology.

It is a legitimate hypothesis that improving sliding properties, which is the purpose of oiling or modification with molybdenum disulfide, may impair polyamide's ability to form effective structural adhesive bonds. In this study, adhesive properties of polyamide PA6 and polyamide modified with molybdenum disulfide $\mathrm{PA} 6-\mathrm{MoS}_{2}$ were analysed. Static shear strength of doubleoverlap adhesive bonds made of the analysed grades of polyamide (connectors) and aluminium alloy EN AW 2024 (overlays) was taken as a measure of adhesive properties. Double-overlap adhesive bonds were chosen to eliminate the effect of bending (typical of lap bonds). The main purpose of the tests and analyses was to compare 
the strength of adhesive bonds for polyamide PA6 (basic), polyamide PA6 - MoS2 and PA6G + oil. A secondary goal was to select a surface preparation technology that would allow maintaining or improving the adhesive properties of the modified polyamide at comparable level to polyamide without modification. Additionally the study covers, the effectiveness of plasma treatment, sol-gel and sandblasting on the energetic properties of the surface layer and bonding effects of basic polyamide, oiled polyamide and polyamide modified with molybdenum disulfide were investigated.

\section{MATERIALS, APPARATUS AND METHODS}

The study used double-overlap samples according to ASTM D3528 [19]. They are particularly dedicated to shear strength testing when joining materials with reduced bending stiffness. Double-overlap samples are also used to test the properties of adhesives in the hardened state, especially the shear stiffness of the adhesive. In the case analysed, polyamide PA6 and polyamide PA6 - $\mathrm{MoS}_{2}$ with EN - AW 2024 aluminium alloy overlays and polyamide PA6 $\mathrm{G}+$ oil were bonded. In the last configuration, the overlays and connectors were made of polyamide. The investigated unmodified polyamide grade PA6 was TECAMID, whereas the modified polyamides were TECAST kind, both manufactured by Ensinger [20, 21, 22]. The samples were bonded with Loctite EA 9392 AERO. The adhesive was applied with a spatula, spread evenly over the connectors and overlays of the bonded elements. The overlays were positioned relative to the connectors using of locating pins. The panels were hardened for 24 hours in a vacuum bag. Panels were hardened for a minimum of 7 days before samples were made. Figure 1 shows the process of panel preparation. The bonded panel contains about 7-8 specimens. The panel is cut into individual samples after seasoning period.

A drawing of a double-overlap sample, with dimensions according to ASTM D3528 is shown in Figure 2. The samples cut using a water abrasive cutting machine, were subjected to measurement inspection to verify that the required dimensional tolerance was maintained. It should be emphasised that keeping sample sizes within tolerance in a series of samples is not an easy matter and requires the use of appropriate technologies. This is not always observed in scientific research, hence the often observed high levels of scatter in terms of strength of the tested series of samples. Consequently, it requires a larger number of samples to be able to estimate the mean value at a defined level of significance. The following connection and overlay surface preparation technologies were used in the experiment:

Sandblasting (abrasive jet machining) with the following parameters: feed pressure 0.07 $\mathrm{MPa}$, head travel speed $50 \mathrm{~mm} / \mathrm{s}$, distance of the head tip from the surface $90 \mathrm{~mm}$, nozzle tip oriented perpendicularly, number of passes 1 , grain: electrocorundum F70.

Atmospheric argon plasma treatment (cold) with the following parameters: plasma power 180 $\mathrm{W}$, plasma head speed $4000 \mathrm{~mm} / \mathrm{min}$, oxygen consumption $0.3 \mathrm{l} / \mathrm{min}$, helium consumption $30 \mathrm{l} /$ $\mathrm{min}$, number of passes 6 . Plasma treatment was applied to a surface using a nozzle, which directed a stream of flowing gas to the surface. The device used for treatment was Atomflo 500 plasma system by Surfx Technologies. The nozzle movement was done in an automated manner using a $\mathrm{CNC}$ router.

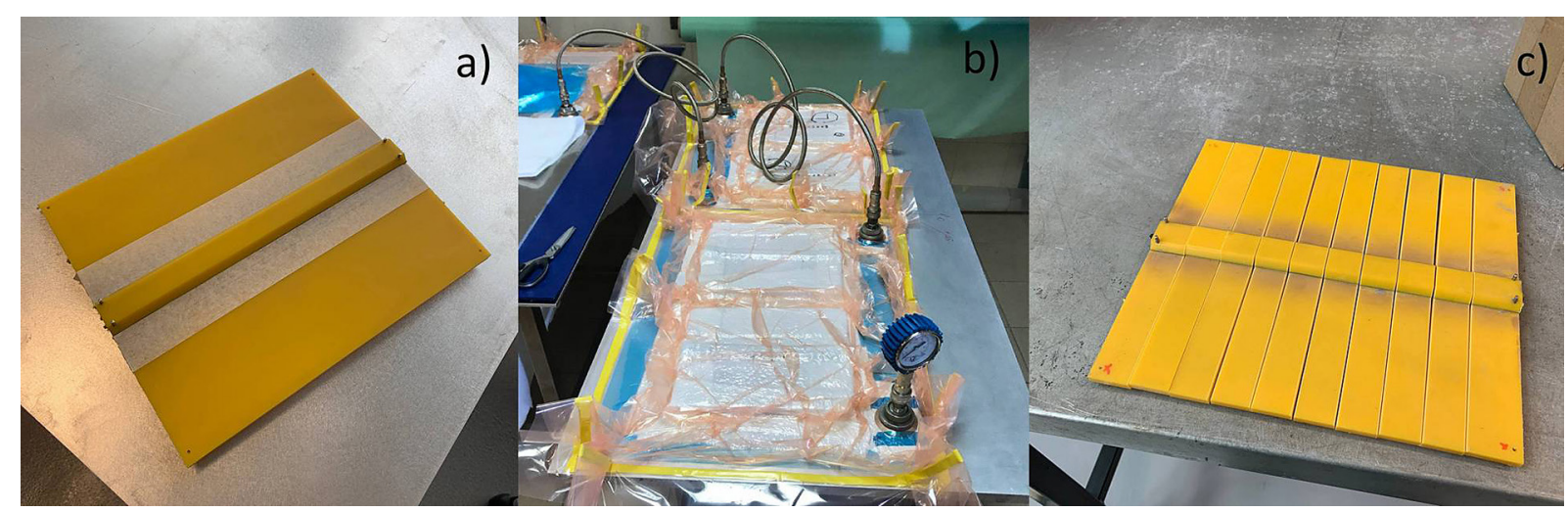

Fig. 1. Preparation of samples in the form of panel, containing about 7-8 individual samples; a) bonded panel prepared for curing, b) curing in the vacuum bag, c) panel cut in the individual samples 

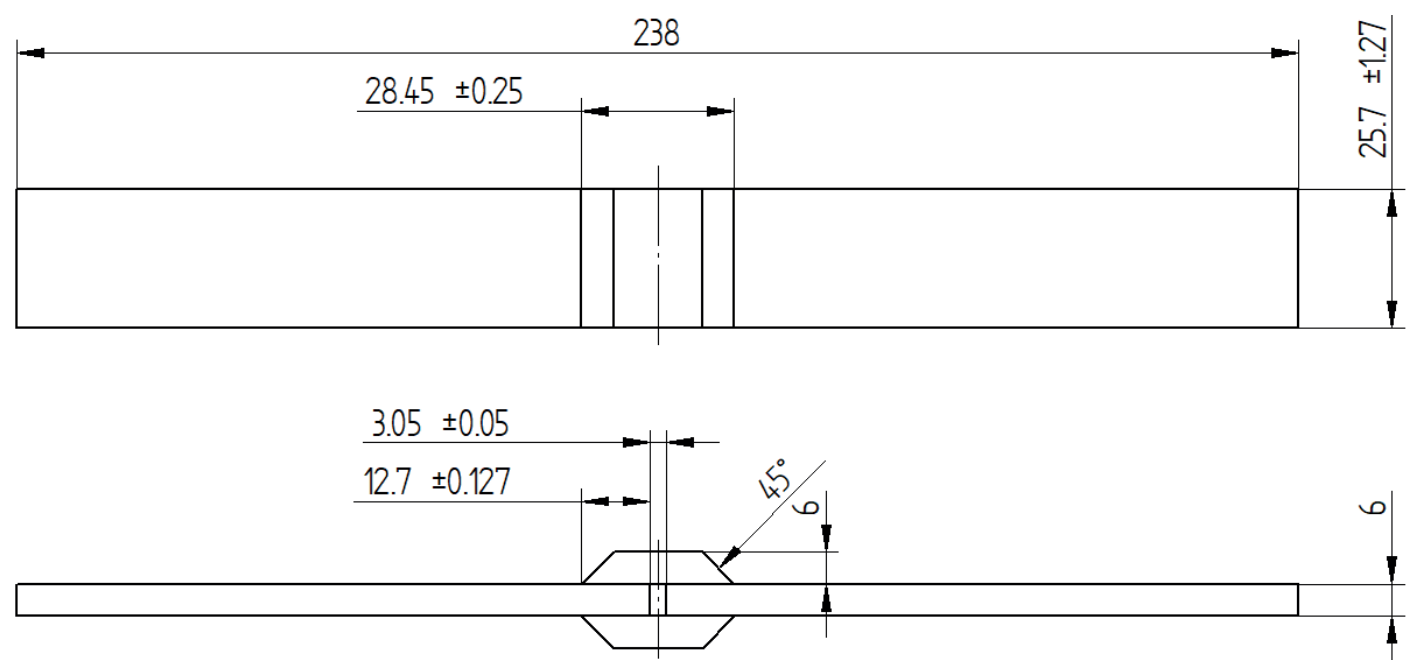

Fig. 2. A double-overlap sample, manufactured in accordance with ASTM D3528

Application of sol-gel primer, according to procedure. The surface was prepared by precleaning with a cleaning cloth and Loctite SF 7063 universal remover. In the next step, the surface was sanded with a Scotch-Brite Heavy Duty Hand Pad 7440, maintaining a $90^{\circ}$ directional change, and cleaned again with the remover. Sol-gel AC-130-2, manufactured by $3 \mathrm{M}$, was prepared and the solution was generously applied with a brush to the surface of the bonded parts. The excess was removed and allowed to dry for a smooth and continuous coating. The bonding was performed keeping the adhesive's shelf life.

The following configurations of surface preparation technologies were tested:

1. Overlays (plasma treatment) - connectors (sandblasting)

2. Overlays (plasma treatment) - connectors (plasma treatment)

3. Overlays (sol gel) - connectors (sandblasting)

4. Overlays (sol gel) - connectors (plasma treatment)

5. Overlays (sandblasting) $\quad-$ connectors (sandblasting)
One of the most important geometrical factors significantly affecting the strength of adhesive bonds is the thickness of the adhesive layer and the uniformity of this thickness in a series of tested samples. In addition, under-bonding may occur in the adhesive zone, which is usually a consequence of hardening shrinkage under insufficient pressure during the process. These under-bonding defects can occur at various locations of the adhesive bond and are detected by defectoscopic testing. An example of a made sample is shown in Figure 3.

The shear strength of the adhesive bonds was performed in accordance with ASTM D3528. The samples were placed in the jaws of the testing machine, clamped and the test was started. The samples were tensioned with a pre-load equal to 10 $\mathrm{N}$. The test was carried out at a traverse speed of $3 \mathrm{~mm} / \mathrm{min}$. In each series, 5 samples were tested. The maximum breaking force was recorded, on the basis of which the shear stress was calculated. Destructive testing was performed on a Zwick Roell Z150 testing machine, shown in Figure 4.

At this point of the research, the obtained results were summarised and compiled. Authors

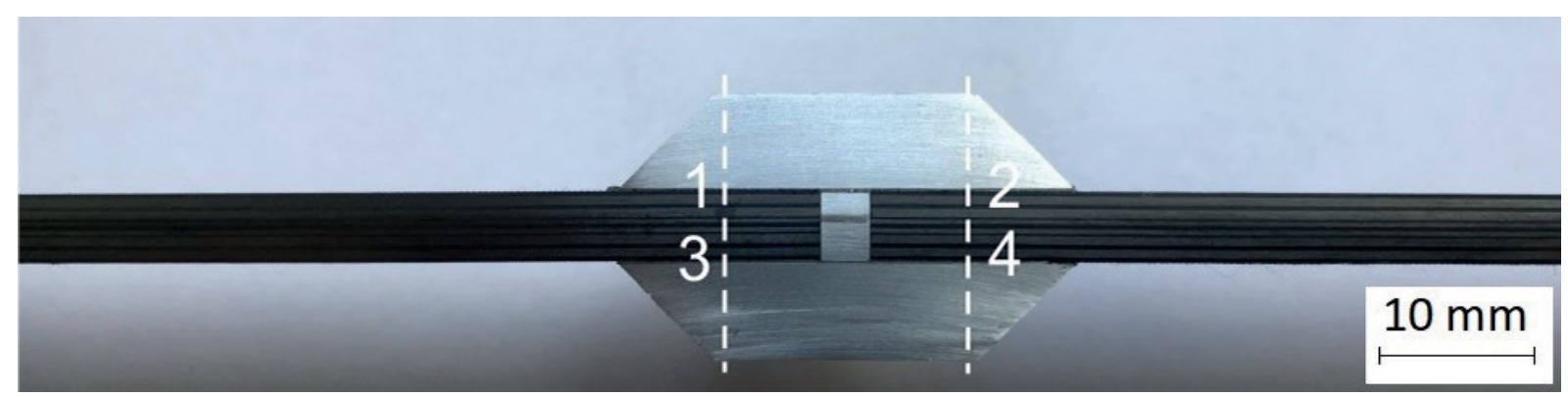

Fig. 3. View of a typical double-overlap sample used in the experiment with indicated four adhesive bonds (1-4) 


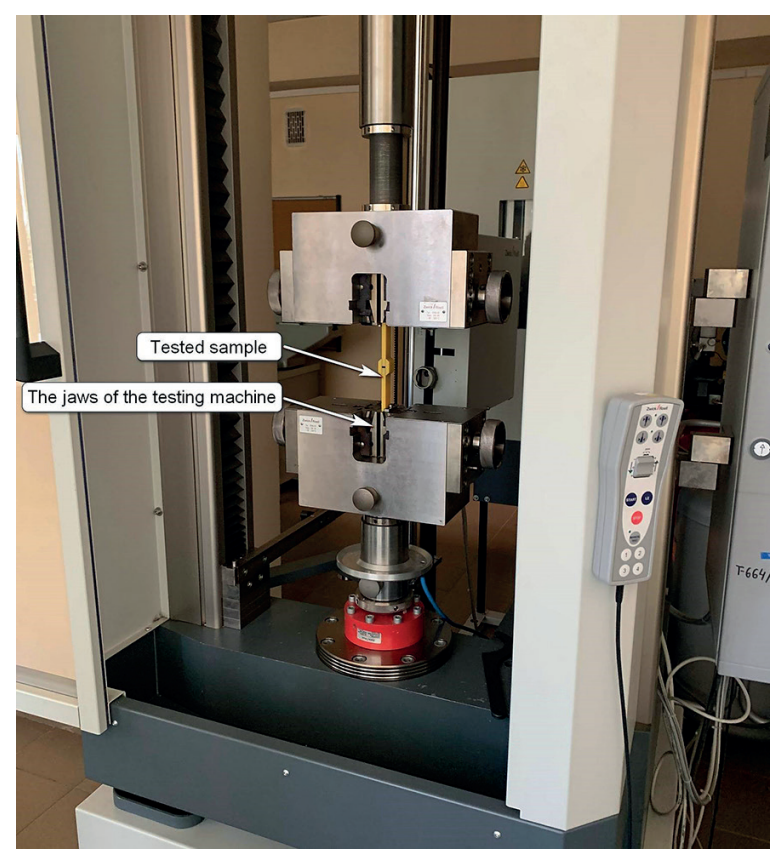

Fig. 4. Testing machine ZWICK/ROELL Z150 with investigated sample mounted in the jaws

verified the results and picked samples prepared with sandblasting, as the ones which achieved the highest shear strength, for further investigation. As one of the goals of the research is to pick the surface treatment which preserves or improves the adhesive properties of the modified polyamides, sandblasted samples were further investigated with measurements of static contact angle and surface roughness. Static contact angle measurement was performed with PGX goniometer using distilled water and diiodomethane. The measurement for each tested surface was repeated 10 times. A single droplet had a volume of $2 \mu$ l. A 3D T8000 RC120-400 Hommel Etamic profilometer was used to measure surface roughness. The measuring was held according to ISO 4287. Measurements of 2D surface roughness parameters were performed within the $4.8 \mathrm{~mm}$ sampling length and repeated 10 times for each of the tested materials and surface preparation methods. Measurements were taken on the area prepared for bonding.

\section{RESULTS}

An important objective in the strength tests was to verify whether modification of polyamide with molybdenum disulfide and oiling, in order to improve sliding properties, significantly alters the adhesive properties of the surface, understood in this study as susceptibility to structural bonding. The results obtained in the axial tensile test of the samples were developed and presented in the form of graphs for Polyamide PA6, Polyamide PA6 $\mathrm{MoS}_{2}$ and Polyamide PA6G + oil are shown in Figure 5.

The results obtained clearly show the positive effect of sandblasting on the strength of PA6AL2024 adhesive bonds in comparison with plasma

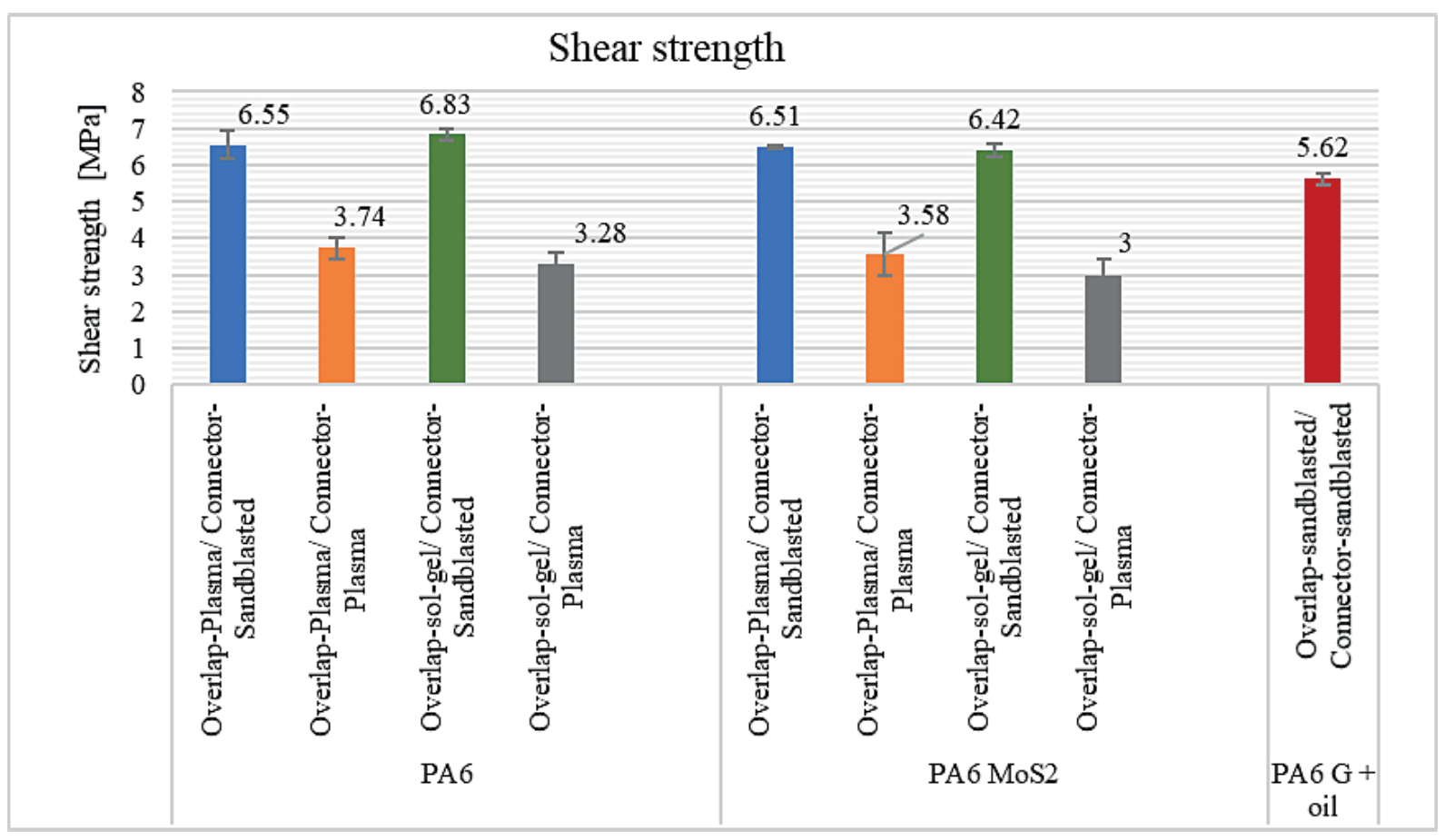

Fig. 5. Comparison of the shear strength of double-overlap samples 


\section{Surface roughness $\mathrm{Ra}$}

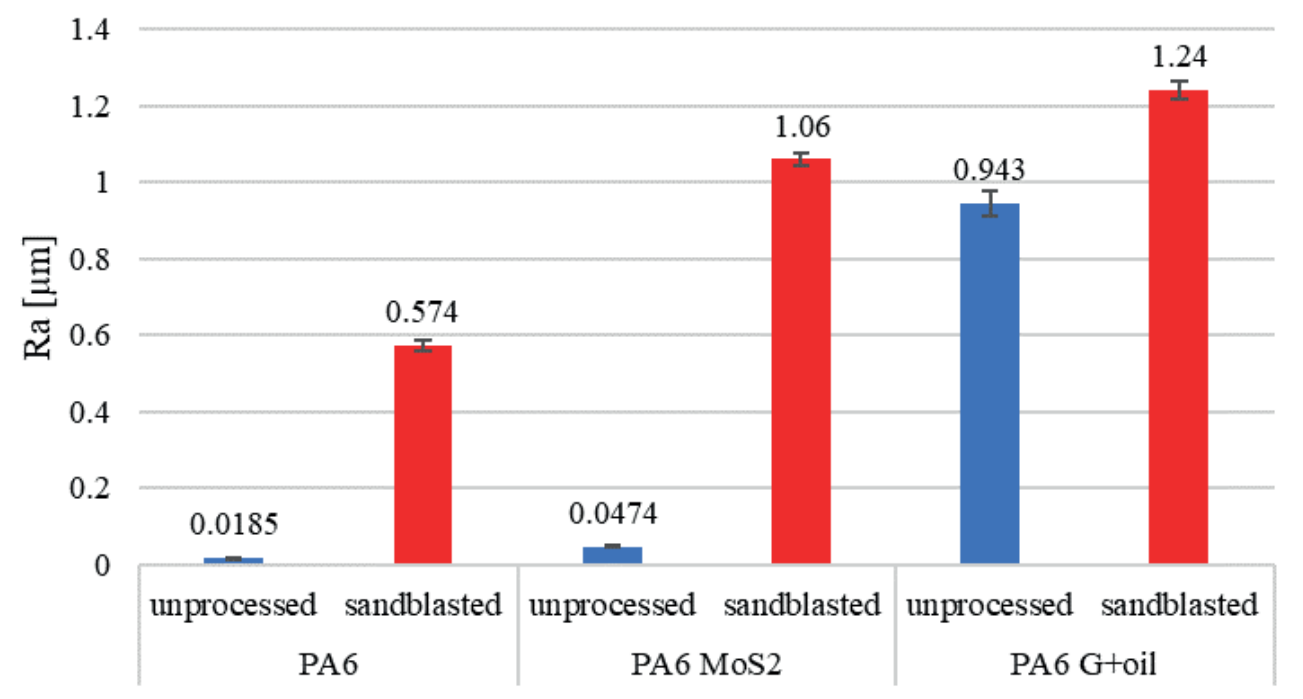

Fig. 6. Surface roughness of PA6, PA6 $\mathrm{MoS}_{2}$ and PA6G+oil joints without surface preparation and after sandblasting

activation. A similar relationship was obtained for polyamide modified with molybdenum disulfide (connectors) and AL2024 (overlays). In this case also, sandblasted connectors showed an advantage over plasma-activated connectors. Such effects were obtained regardless of whether the overlays had a sol-gel coating or were plasma-activated. For polyamide $\mathrm{PA} 6 \mathrm{G}+$ oil both the connectors and the overlays were made of polyamide, with sandblasting as the only surface preparation technology. In this case, the results made it possible to assess the effect of sandblasting on the strength of an adhesive bond that is homogeneous in terms of materials.

A lower strength values were achieved for samples prepared with plasma treatment in comparison to these prepared with sandblasting. Since the research has a specifically industrial application, authors decided to investigate sandblasted samples only. In order to compare the quality of the surfaces prepared for bonding, roughness measurements and contact angle measurements were performed for PA6, PA6 $\mathrm{MoS}_{2}$ and PA6G + oil materials to determine the value of surface free energy. Measurements were performed on the untreated and sandblasted surface. The graph in Figure 6, shows the roughness parameter $\mathrm{Ra}$ for all the materials tested. Ra parameter was used for comparison because prepared surface area is small and uniformly prepared, so the parameter will well map the surface condition and the presented results are focused on the industrial applications.

Sandblasting in each case "developed" the polyamide surface geometrically. The significant difference in Ra value of oiled polyamide in the untreated state compared to PA6 and PA6 $\mathrm{MoS}_{2}$ is puzzling. This implies a significant influence of technology and tooling condition at the polyamide plates manufacturing stage.

The surface free energy (SEP) was also compared. A summary of the surface free energy results calculated by the Owens - Wendt method is provided in the graph shown in Figure 7.

The value of surface free energy was calculated from the following relationships $(1,2,3)$.

$$
\gamma_{s}=\gamma_{s}^{d}+\gamma_{s}^{p}
$$

$\left(\gamma_{s}^{d}\right)^{0,5}=\frac{\gamma_{d}\left(\cos \Theta_{d}+1\right)-\sqrt{\frac{\gamma_{d}^{p}}{\gamma_{w}^{p}} \gamma_{w}\left(\cos \Theta_{w}+1\right)}}{2\left(\sqrt{\gamma_{d}^{d}}-\sqrt{\gamma_{d}^{p} \frac{\gamma_{w}^{d}}{\gamma_{w}^{p}}}\right)}$

$$
\left(\gamma_{s}^{p}\right)^{0,5}=\frac{\gamma_{w}\left(\cos \Theta_{w}+1\right)-2 \sqrt{\gamma_{s}^{d} \gamma_{w}^{d}}}{2 \sqrt{\gamma_{w}^{p}}}
$$

where: $\gamma_{\mathrm{S}}-$ SEP of tested surface; $\gamma_{\mathrm{S}}{ }^{\mathrm{d}}-$ dispersive component; $\gamma_{S}{ }^{p}$ - polar component; $\theta$ - contact angle between tested surface and test liquid; $\gamma_{\mathrm{w}}-$ free surface energy of water; $\gamma_{\mathrm{w}}^{\mathrm{d}}$ - dispersive component of SEP of water; $\gamma_{\mathrm{w}}{ }^{\mathrm{p}}$ - polar component of SEP of water; $\gamma_{d}-$ SEP of diiodomethane; $\gamma_{d}{ }^{d}-$ dispersive component of SEP of diiodomethane; $\gamma_{d}^{p}-$ polar component of SEP of diiodomethane. 


\section{Surface free energy}

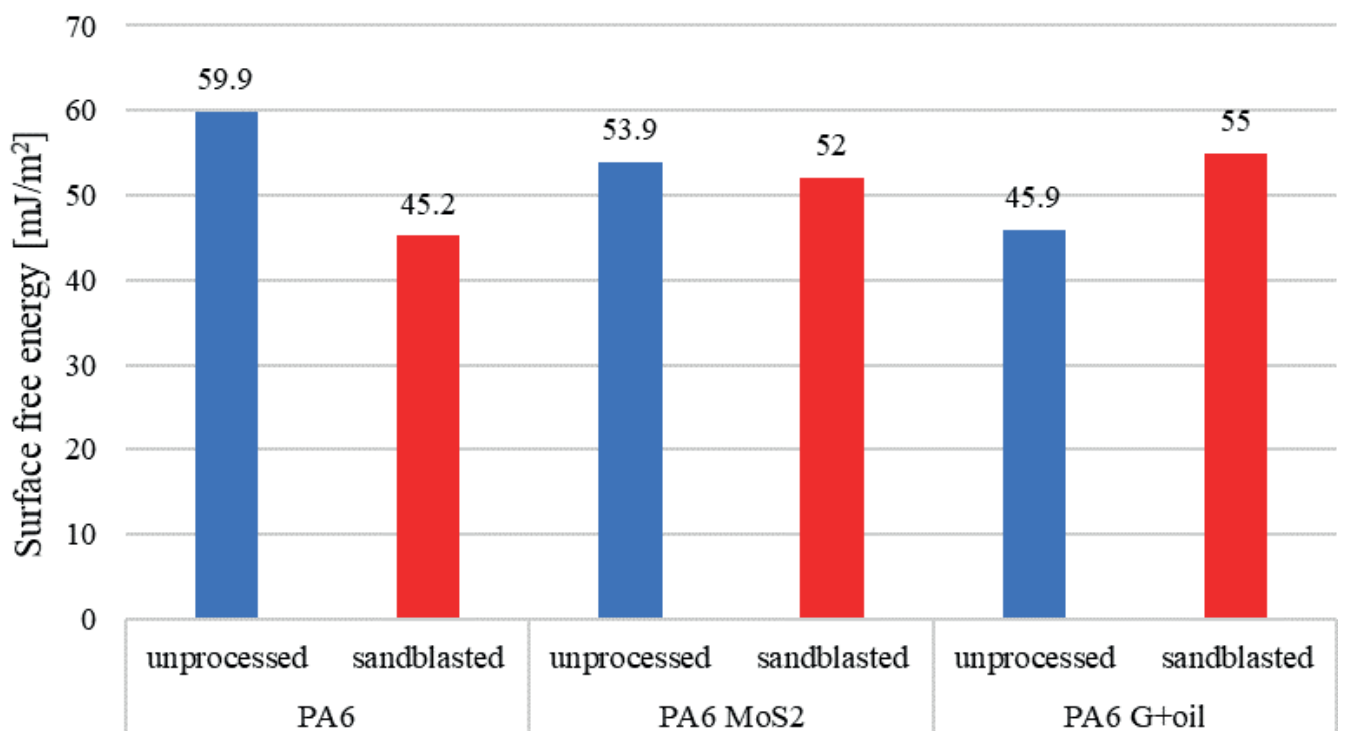

Fig. 7. Free surface energy of PA6, PA6 $\mathrm{MoS}_{2}$ and PA6G+oil without surface preparation and after sandblasting

A relatively small improvement in surface free energy after sandblasting (only in the case of oiled polyamide) in comparison with the untreated state is noticeable. In other cases, a decrease in SEP was observed. It should be noted, however, that a high SEP value is a necessary but not sufficient condition for a high strength adhesive bond. It may be that the physicalsorption layer covering the surface of the slab, as a result of technological history, has a high SEP value, but it is a poorly bonded layer to the substrate and may constitute a weakening boundary layer.

\section{DISCUSSION}

When analysing the results of the tests, one can notice significantly higher values of the strength of adhesive bonds of the samples whose connector surfaces were sandblasted, in comparison to those whose connector surfaces were plasma-treated. Samples with sandblasted connectors are characterised by $81.8 \%$ higher shear strength than samples with plasma-treated connectors and overlays and $114 \%$ higher shear strength than samples with sol-gel overlays and plasma-coated connectors. These differences are similar for both bonded materials: PA6 and PA6 $\mathrm{MoS}_{2}$ It can be concluded that surface preparation using sandblasting gives much better results (in terms of joint strength) than plasma and sol-gel methods. The results of samples prepared by combined plasma and sol-gel methods are much worse. The use of plasma method for the preparation of both overlays and connectors allowed to reach higher shear strengths, respectively by $14.02 \%$ for polyamide PA6 and by $19.33 \%$ for polyamide PA6 $\mathrm{MoS}_{2}$, as compared to samples with overlays treated by solgel method and connectors treated by plasma.

Also it is important to compare the effect of modifying polyamide with friction reducing agents on the adhesive properties measured as the strength of the adhesive bond shear strength. Samples in the configuration of overlays treated with plasma / connectors sandblasted made of PA6 $\mathrm{MoS}_{2}$ achieved marginally lower strengths, by an average of $0.6 \%$, compared to those with PA6. Compared to the samples made of PA6 G + oil, a 14.2\% lower strength was observed. For samples in the sol-gel overlay / connectors sandblasted configuration, compared to samples made of PA6, the results achieved by samples made of PA6 $\mathrm{MoS}_{2}$ are on average $6 \%$ lower, for PA6 G + oil on average $17.7 \%$ lower. In the case of surface preparation by sol-gel and plasma methods, a decrease in strength by $4.28 \%$ on average was observed in comparison with samples with overlays and connectors treated with plasma. An average decrease of $8.54 \%$ was observed for PA6 $\mathrm{MoS}_{2}$ samples with overlays treated by sol-gel method and connectors treated by plasma spraying in comparison with samples treated using the same method made of PA6 material. The high value of standard deviation should also be noted, reaching up to $30 \%$ in the case of samples with overlays treated by plasma and sol-gel methods. Based on the results obtained, it can be concluded that the 
method of polyamide surface activation by plasma treatment is unsatisfactory as a method preparing the surface for bonding. When sandblasting is used on connectors or overlays, the standard deviation is at an acceptable level. Comparing the roughness value with the results of strength tests, attention should be paid to similar values of surface roughness of sandblasted samples of polyamide with friction reducing agents. Although the use of the same sandblasting parameters, for all tested materials, the surfaces of the modified polyamides are characterized by a higher roughness, compared to the unmodified polyamide. The difference in surface roughness between unmodified and modified polyamide can be addressed to the reduced hardness of the modified material itself. Despite the similar surface roughness values of PA6G + oil and PA6 $\mathrm{MoS}_{2}$, lower strength of the adhesive bond of samples made of PA6G + oil was obtained. The reduction in strength can be attributed to the presence of friction reducing agents.

The obtained results can only be indirectly compared to the studies presented in the literature due to the lack of studies on adhesive bonds of polyamide modified by adding friction reducing agents. It can be concluded that in the case of applying plasma treatment on modified polyamide surface with friction reducing agents, slightly worse results are obtained than in the case of activating the surface of the polyamide without modification. For samples with a surface prepared for adhesive bonding by plasma treatment, the obtained results were similar to the research presented in the literature [14]. Slightly worse results of the shear strength of samples with the surface prepared by plasma treatment can be observed in the case of breaking single-overlap samples, which may be mainly caused by the non-axial load [18]. In case of comparison of the samples prepared by plasma treatment, from PA6 and PA6 MoS 2 material with the literature research, it should be considered that the results presented in the article are equivalent, regardless of the introduction of friction reducing additives. Sandblasting should be considered as the leading method of surface preparation. In favour of plasma treatment, the repeatability of the process parameters, as well as the ease and cleanliness of the treatment, should be emphasized.

Achieved research results allow for the elimination of additional connecting elements, e.g. bolts, rivets and at the same time weight reduction of the structure. Maintaining good adhesive properties despite the introduction of lubricants to the polyamide, can be attributed to one of the theories of adhesion, to be precise, mechanical adhesion, which consists in mechanically anchoring the adhesive in the micropores of the base material. This is also confirmed by the surface free energy values shown in Figure 7. They are also at comparable levels. The surface roughness was also examined. It is worth noting that roughness of the untreated (raw) polyamide surface increases with the introduction of friction reducing substances into its composition.

\section{CONCLUSIONS}

Basing on the presented results the following conclusions can be formulated:

- The addition of friction reducing agents to polyamide does not significantly impair the adhesive properties of the materials presented. This is an interesting piece of information of a utilitarian nature as well. It shows that in machines and devices using modified polyamide, it is possible to use adhesive bonds in friction nodes with effects comparable to those of bonding "pure" polyamide.

- A slight decrease in the shear strength of modified versus unmodified polyamides can be explained by higher surface roughness of modified polyamide. This observation shows that the influence of surface roughness, in the comparative tests, on the strength of adhesive joints of unmodified and modified polyamides is complex. The modified polyamides due to its reduced hardness are more susceptible to shaping the surface by sandblasting. Correction of sandblasting parameters could potentially further improve the strength of the joints made.

- The test results prove that the introduction of friction-reducing agents into the polyamide composition is not a factor that deteriorates the adhesive properties. It appears that in case of polyamide adhesive bonding, the strength of the bond can be attributed to mechanical adhesion, which can be improved by proper surface roughening. It should be emphasized that the preparation of the surface is still the most important factor determining the quality of the connections made.

- The proper preparation of polyamide surface by sandblasting, independently on its modification with friction reducing agents, can ensure satisfactory results of adhesive bonding strength for the materials studied. 


\section{Acknowledgements}

This work was financed from the funds of the Ministry of Education and Science by Agreement No. DNK/SP/513880/2021 of 22 December 2021, the project " $14^{\text {th }}$ School of Machining and the $43^{\text {rd }}$ Scientific School of Abrasive Machining", under the programme "Perfect Science".

\section{REFERENCES}

1. Gruin I. Materiały Polimerowe. Polish Scientific Publishers PWN; 2003.

2. Drobny J.G. Handbook of Thermoplastic Elastomers. Elsevier; 2014.

3. Mishra M. Encyclopedia of Polymer Applications. CRC Press; 2018; 3.

4. Hussain C.M. Handbook of Polymer Nanocomposites for Industrial Applications. Elsevier; 2020.

5. Kinloch A.J. Adhesion and Adhesives Science and Technology. Springer Science \& Business Media; Berlin, Germany; 1987.

6. Kuczmaszewski J. Fundamentals of Metal-Metal Adhesive Joint Design. Lublin University of Technology. Polish Academy of Sciences; 2006.

7. Mandolfino C., Lertora E., Gambaro C., Pizzorni M. Durability of polyamide bonded joints: influence of surface pre-treatment. International Journal of Adhesion and Adhesives. 2018; 86: 123-130.

8. Primc G. Surface Modification of Polyamides by Gaseous Plasma - Review and Scientific Challenges. Polymers. 2020; 12(12).

9. Kłonica M., Kuczmaszewski J., Kwiatkowski M.P., Ozonek J. Polyamide 6 surface layer following ozone treatment. Int. J. of Adhesion\&Adhesives. 2016; 64: 179-187.

10. Károly Z., Kalácska G., Sukumaran J., Fauconnier D. Effect of Atmospheric Cold Plasma Treatment on the Adhesion and Tribological Properties of Polyamide 66 and Poly(Tetrafluoroethylene). Materials. 2019; 12(4): 658.

11. Ebnesajjad S. Introduction and adhesion theories. Handbook of Adhesives and Surface Preparation Technology, Applications and Manufacturing. Elsevier; 2011.
12. Krasinskyi V., Suberlyak O., Kochubei V., Klym Y., Zemke V., Jachowicz T. Effect of Small Additives of Polyamide Modified by Polyvinylpyrrolidone and Montmorillonite on Polypropylene Technological Properties and Heat Resistance. Advances in Science and Technology Research Journal. 2018; 12(2): 83-88. DOI: 10.12913/22998624/90924.

13. Krasinskyi V., Suberlyak O., Kochubei V., Jachowicz T., Dulebova L., Zemke V. Nanocomposites Based on Polyamide and Montmorillonite Obtained from a Solution. Advances in Science and Technology Research Journal. 2020; 14(3): 192-198. DOI: $10.12913 / 22998624 / 122297$.

14. Mandolfino C., Lertora E., Gambaro C. Influence of cold plasma treatment parameters on the mechanical properties of polyamide homogeneous bonded joints. Surface and Coatings Technology. 2017; 313(15): 222-229.

15. Musiari F., Moroni F. Experimental Study of the Influence of the Surface Preparation on the Fatigue Behavior of Polyamide Single Lap Joints. Materials. 2021; 14(4): 1008.

16. Lim S.J., Cheon J., Kim M. Effect of laser surface treatments on a thermoplastic PA 6/carbon composite to enhance the bonding strength. Composites Part A. Applied Science and Manufacturing. 2020; 137(105989).

17. Borcia G., Dumitrascu T.N., Popa G. Influence of helium-dielectric barrier discharge treatments on the adhesion properties of polyamide- 6 surfaces, Surface and Coatings Technology. 2005; 197(2-3): 316-321.

18. Károly Z., Kalácska G., Zsidai L., Mohai M., and Klébert S. Improvement of Adhesion Properties of Polyamide 6 and Polyoxymethylene-Copolymer by Atmospheric Cold Plasma Treatment. Polymers. 2018; 10(12).

19. ASTM D3528 - 96. Standard Test Method for Strength Properties of Double Lap Shear Adhesive Joints by Tension Loading. 2016.

20. Ensinger PA6. https://www.ensingerplastics.com/ en-gb/shapes/products/pa6-tecamid-6-natural (accessed Sept. 24 2021)

21. Ensinger PA6 $\mathrm{MoS}_{2}$. https://www.ensingerplastics. com/en-gb/shapes/products/pa6-tecamid-6-moblack (accessed Sept. 24 2021)

22. Ensinger PA6G + oil. https://www.ensingerplastics.com/en-gb/shapes/products/pa6-tecast-1-yellow (accessed Sept. 24 2021) 